\title{
Exploring the Gait and Stability of Passengers at the Moment they get off an Urban Railway Train by Laboratory Experiments
}

\author{
Sebastian Seriani ${ }^{1}$. Cristobal Erenchun ${ }^{2}$. Felipe Palma ${ }^{3}$ \\ ${ }^{1}$ Escuela de Ingeniería de Construcción y Transporte, Pontificia Universidad Católica \\ de Valparaíso, Valparaíso, Chile, \\ E-mail: sebastian.seriani@pucv.cl \\ 2 Universidad de los Andes, Faculty of Engineering and Applied Sciences, Santiago, Chile, \\ E-mail: cerenchun@miuandes.cl \\ ${ }^{3}$ Universidad de los Andes, School of Kinesiology, Santiago, Chile, \\ E-mail: fpalma@uandes.cl
}

Received: 2 July 2021 / Last revision received: 29 September 2021 / Accepted: 16 December 2021 DOI: $10.17815 / C D .2021 .115$

\begin{abstract}
The Santiago of Chile subway system is nowadays one of the most used means of transportation in the city, therefore many passengers with reduced mobility prefer it. However, in the subway lines, we can find different vertical gaps that are generated between the train and the platform. These vertical gaps makes it difficult for passengers with reduced mobility to get on and off the train, by generating a change in their gait, affecting their stability. This stability can be represented by different variables such as the rejection and damping force, the travel ranges of each step component, and the area generated by these travel ranges. The objective of this paper is to study, experimentally, the effect of vertical gaps on the gait and stability of passengers with reduced mobility in the train-platform space of subway stations. For this purpose, the construction of fullscale experiments representing the train-platform transition was carried out at the Human Dynamics Laboratory of the Universidad de los Andes. To obtain the data, a Bertec force plate and Bertec Acquire 4 software were used, which allows, to obtain the force in the zaxis and the pressure centers in the $\mathrm{x}$-axis and y-axis. The results show that the higher the vertical gap, the higher the instability in passengers with reduced mobility. In addition, it was also observed how passengers with reduced mobility change their gait strategy when having to face vertical gaps greater than $11 \mathrm{~cm}$, since they changed the angle of inclination
\end{abstract}


with which people position their foot when descending, to be able to do the process with greater stability. It is hoped that future experiments will expand the scope of this type of study, by implementing more instrumentation and a larger number of participants.

Keywords Passenger with reduced mobility $\cdot$ gait $\cdot$ stability $\cdot$ train-platform interface vertical gap $\cdot$ experiments

\section{Introduction}

As in other cities around the world, in Santiago de Chile, the most important public transportation is the urban train or subway. This system is the main skeleton that connects all the networks of the capital city, with seven lines and connections to other public transportation networks, such as suburban buses and the Metropolitan Mobility Network [1]. This at the same time presents an essential characteristic, which is to have universal accessibility, achieving that passengers with reduced mobility can use it without problems, without any restriction, and autonomously $[2,3]$. Universal accessibility should be understood as what is stipulated in the Universal Accessibility Law, a regulatory context in which it should govern, inspire, and achieve: "Moving towards an inclusive and modern country, which grants everyone equal opportunities to fulfill personal goals and aspirations: a challenge to architecture, urbanism, public programs and projects to incorporate the necessary conditions so that our cities, buildings, squares, and parks have possibilities of access and use for all their inhabitants" [2].

This quote explains in broad terms, that Chile is giving an important impulse to the design of cities to comply with all current regulations. Therefore, as it is reported by Tyler [4] the different public transport systems have the challenge of adapting to universal accessibility, so that passengers can not only access it but also use it. Accessibility today is a broad and relative term, essential both to achieve effective equality of opportunity for all people and to optimize the design of an environment [5,6]. However, different types of designs have been used to improve accessibility in public transport: there are accessibility designs that are intended for passengers with reduced mobility. For example, in Santiago subway stations, elevators or ramps are used as an aid for passengers with reduced mobility who require a wheelchair as a tool to move, but the use of stairs with handrails or lower steps are solutions that have been implemented to provide accessibility. All designs must improve the quality of life [7].

In urban trains, accessibility problems are concentrated in the train-platform interface, which presents higher risks of accidents for passengers with reduced mobility [8]. In the case of the subway in Santiago de Chile (see Fig. 1), a rubber material has been used as a filler to reduce the horizontal gap between the platform and train. On the other hand, to reduce the vertical gap some metro stations have introduced raised platforms. As an example, Green Park station on the London Underground has a raised platform that extends on a part of the platform and has a total length of $27 \mathrm{~m}$, this element covers the second and third carriage and a total of four doors (two double and two single). Despite different measures to improve universal accessibility at such interface in the Santiago 
Metro [2,3], the solutions are limited to different factors such as platform design (e.g. curved platforms), train doors (e.g. number and width of doors), and train floor (e.g. material), therefore it cannot be implemented in all stations. As a consequence, some stations will still have a vertical gap, which is a fixed value considering the train type and interface design at each station [9]. To delve into vertical gap designs, studies by Fernandez et al. $[10,11]$ sought to simulate flows in which participants board and alight from three different vertical gaps: $0 \mathrm{~cm}, 15 \mathrm{~cm}$, and $30 \mathrm{~cm}$. The results show a better design would be within the range of $0 \mathrm{~cm}$ to $15 \mathrm{~cm}$. This opens up a range in which this study seeks to work and see how the gait process behaves for passengers with reduced mobility.
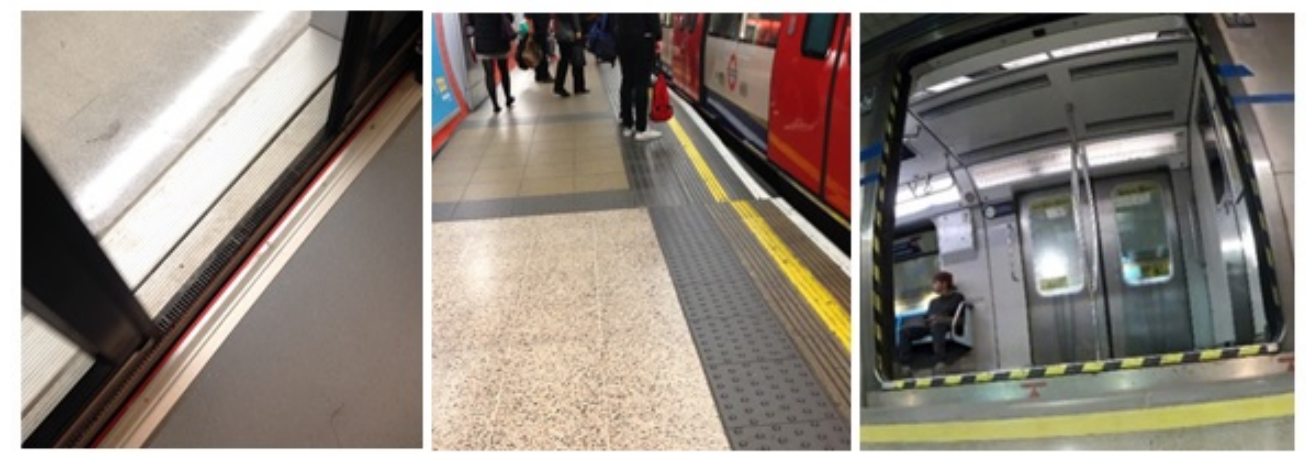

Figure 1 Vertical gaps in subway stations: rubber material (left), elevated platform (center), existing vertical gap (right)

To improve universal accessibility at the train-platform interface, it is necessary to study how vertical gaps affect the gait and stability of passengers with reduced mobility in the process of alighting the train, which is the aim of this research. To this objective, an interdisciplinary methodology is proposed, combining knowledge of transportation and kinesiology through instrumentation and study methods. The specific objectives are: (a) to define variables that affect the gait and stability of passengers when facing a vertical gap, (b) to define a method to measure those variables, (c) to simulate at the Human Dynamics Laboratory of the Universidad de los Andes (HDL) passengers alighting a train in a full-scale experiment, and (d) to analyze the data obtained in the experiment to see how the gait and stability of a passenger with reduced varies in different vertical gaps.

This article is structured in five sections, starting with the introduction. In section two, existing studies related to the gait and stability are presented. In section three, the method is explained based on laboratory experiments. In section four, the results are presented followed by a discussion and conclusions in section five.

\section{Existing studies on vertical gaps and stability}

The locomotor task is quite demanding for passengers with reduced mobility, especially considering their mobility restrictions in their lower extremities. Facing a vertical gap can 
be very difficult, so there is an urgent need for a way, form, and solution that does not demand too much physical effort, and which would be a significant help in the quality of life of people with physical impairments [2,3].

Different laboratory experiments have been performed to study vertical gaps. According to Holloway et al. [12] greater vertical gaps produce an obstacle for those passengers boarding and alighting, especially for passengers with reduced mobility. In the same line of research, Daamen et al. [13] reported that steps can influence the flow of passengers boarding and alighting. The authors found that passengers could overpass a vertical gap of $2 \mathrm{~cm}$, however if the vertical gap reached $5 \mathrm{~cm}$ one-third of wheelchair passengers presented problems. If the vertical gap was equal to $10 \mathrm{~cm}$, most passengers presented difficulties, and therefore the vertical gap should be reduced to their minimum to improve accessibility. Some studies performed by Atkins [14] recommend that the sum between the horizontal and the vertical gap should not exceed $300 \mathrm{~mm}$, and that an optimum value for design would be $200 \mathrm{~mm}$. If the vertical gap is greater than $50 \mathrm{~mm}$ and the horizontal gap greater than $75 \mathrm{~mm}$, then a boarding device is needed for passengers with restricted mobility (e.g. wheelchairs). If these values are not in place along the complete platform, Tyler et al. [15] propose to build platform humps, by which only a part of the platform is raised to be level with the vehicle. Moreover, Seriani et al. [9] suggest that vertical gaps should be lower than $5 \mathrm{~cm}$ (and ideally lower than $2 \mathrm{~cm}$ ) to achieve accessibility. In summary, the authors suggest five ranges for the vertical gap. A vertical gap between $0 \mathrm{~cm}$ and $2 \mathrm{~cm}$ is accessible for all users, however if the vertical gap is between $2 \mathrm{~cm}$ and $5 \mathrm{~cm}$, some passengers with reduced mobility will present problems when boarding or alighting the train. In the case of a vertical gap between $5 \mathrm{~cm}$ and $10 \mathrm{~cm}$, most of passengers with reduced mobility will report difficulties, perceiving pain or discomfort. If the vertical gap is greater than $10 \mathrm{~cm}$, it is inaccessible for all users.

Regaining the locomotion, vertical gaps are a key factor in patient returning home after an injury [16]. It can be identified that the gait when passing a vertical gap, could be separated in ascending and descending, which is a significantly complex task for passengers with reduced mobility. As reported by Hashish et al. [17] this task demands physical effort and in turn, the use of some motor capacity, being a potential cause of balance loss and falls. In presence of vertical gaps, Lee and Chou [18] and Bhatt et al. [19], define the gait as the sequence of foot movements by which a person moves forward. The first phase is the cushioning, which corresponds to when the foot makes contact with the ground, taking all the load of the body on the ankle of this foot. The second phase is the rejection, which is defined as the abandonment of this foot from the ground, instance in which the last part to lose contact corresponds to the toes. To study this process, the displacement of the center of mass, which represents the degree of body sway in different directions, must be analyzed. The center of pressure is also important, it refers to the displacement, calculated by the changes in position from the point of ground reaction forces, representing the gait strategy and the stability of the body. Dynamic stability can be calculated as a parameter that simultaneously considers the center of mass and its instantaneous velocity, making it an efficient way to measure losses of balance and falls. In addition, the angle of inclination of the foot in two planes, the anteroposterior plane (inclination angle $\alpha$ ), and lateral midplane (inclination angle $\beta$ ), are two parameters very 


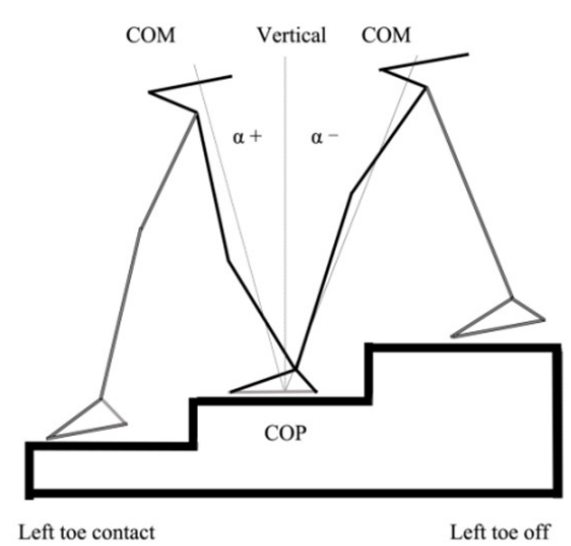

(a)

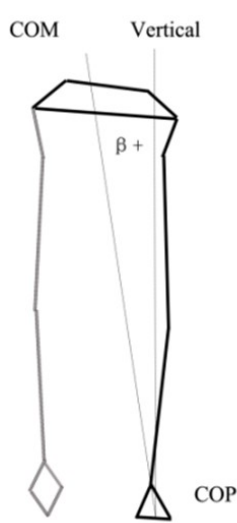

(b)

Figure 2 a) Graphical representation of the right foot when stepping down, considering the center of mass $(\mathrm{COM})$, center of pressure (COP) and inclination angle $\alpha$. b) The lateral midplane considering the inclination angle $\beta$

difficult to measure in real-time but are directly related to instability (see Fig. 2 reported by Song et al. [20]). On the other hand, the force plate is an instrument to measure the ground reaction forces generated by a body passing or standing on it. They are generally used to quantify balance, gait, and other biomechanical parameters. This plate is complemented by VICON cameras, which are used as an instrument to record the movements of people three-dimensionally using reflective metal pins, generally with medical and sports applications.

Based on the above definitions, Song et al. [20] analyzed how people descend a step. The results showed that the higher the complexity of the cognitive or manual task performed when descending a stair, the lower the step velocity, but it did not affect dynamic stability or the angle of inclination. This is because it was believed that gait occurs unconsciously and does not involve higher brain functions. In consequence, the parallel cognitive and manual tasks require additional resources, demonstrating the danger of performing parallel tasks while descending steps. It is also important to note how carrying external loads affects when descending or ascending a step. One foot supports the weight of the whole body, the other foot advances to the next step, generating inequality in the load and consequently an increase in the lateral half velocity of the center of pressure [21]. Also a greater displacement of the center of pressure is reported during the initiation of the step when carrying external loads [22]. On the other hand, a study by Wang and Gillette [23] sought to investigate the effect of symmetric loading and external asymmetric loading on the lower extremities during descent and ascent. The results indicate that carrying a load asymmetrically is more damaging to stability, followed by symmetrical loading. In both cases, this parameter was more impaired during the descent and remained relatively normal during the ascent. 
Being able to measure stability is one of the most important characteristics which helps people to intuit falls and to complete secondary actions while in displacement. According to Huang and Brown [24], a way to measure stability is by obtaining the displacement of the center of pressure. Obtaining the center of pressure is achieved by using a force plate, which provides a point in millimeters of where the center of pressure is located on the plate. In addition, this instrument places an imaginary Cartesian axis where the origin is (right in the center of the plate). The use of the plate is technical related to the quantification of the postural sway or lateral medial plane motion of a standing person. It helps to measure the actual sway of each foot or the sway of the whole body. Lafon et al. [25] reported that the center of pressure displacements are one of the most common measures of body sway, in which a greater sway means greater instability, considered an indication of an impaired postural control system. This is based on many experiments on aging, and pathological conditions that have indeed observed a greater influence in those situations. The center of pressure displacement value depends on where the person steps on the force plate. A typical procedure in the analysis is to remove the mean value from the data, as this is generally the length of the foot, generating the presence of a trend. While it could be argued that the trend itself could provide valuable information about the signal [26], the problem is that most measures used must assume that the signal is stationary. A signal is stationary if its statistical properties (such as mean and variance) do not change over time. Therefore, to remove the trend from the data it may be necessary in certain cases to apply a high-pass filter to the data with a cutoff frequency related to the period of data acquisition [27]. On the other hand, the mean speed expresses the average speed of the center of pressure displacement, calculated simply as the total trajectory variable (the total displacement) divided by the total period. The resulting average velocity is the speed calculated in vector form considering each direction, i.e., the square root of the sum of the squared velocity of the anteroposterior plane direction and the lateral midplane direction. Another way to measure postural sway is to calculate the area encompassed by the center of pressure data in the anteroposterior plane and lateral midplane using a curve in a plane (e.g., a circle or ellipse) or a polygon (e.g., a rectangle). A common method for this is to calculate a prediction ellipse, which is found by fitting an ellipse to the data using concepts from the statistical procedure known as principal component analysis. A 95\% prediction ellipse is a prediction interval for the center of pressure data such that there is a 95\% probability that a new observation will fall within the ellipse. From the interpretation of the center of pressure, it can be said that the greater the area of displacement during a given time, the greater the instability and also the sway (lateral mean plane motion) of the line left by the center of pressure displacement of a person moving straight ahead. The less straight this line is, the greater will be its instability. In conclusion, the bibliographic information indicates that given the literature presented and despite the advances found to date on the gait and stability, a study is required to explore the effect on these variables for different vertical gaps at the train-platform interface, which is the main objective of this paper. 


\section{Method}

\subsection{Experimental design}

Measurements were made on the seven lines of the subway in Santiago, choosing three stations from each line, giving a sample of 21 stations (corresponding to $15 \%$ of the network). Field data were collected from each station in both directions during the week of December 4, 2019. Tab. 1 shows the vertical gaps observed by this study, the stations are grouped by their corresponding line, and each station is divided into Direction 1 and Direction 2, corresponding to each platform, respectively. The vertical gaps are in a range of values between 0 and $12.5 \mathrm{~cm}$. In the case of Quilin station the platform is curved, and therefore two points of measurement were reported: at the end of the train and in the middle of the train. The case of $12.5 \mathrm{~cm}$ corresponds to line 2 (L2), where older trains are used. In the case of line 3 (L3) and line 6 (L6) new trains have platform door technology, and the train runs without a driver (automated). To measure the vertical gap at each station, direct measuring elements (e.g., a measuring rod) were used when the train stopped near the platform. The most critical door of a train was selected, understood as the door that is closer to the exit or entrance of the platform, and therefore the one most likely to be used by passengers with reduced mobility, since this type of passenger tends to become fatigued when traveling on long journeys as reported by Tyler [4].

To conduct the experiment, a full-scale pilot had to be built at the Human Dynamics Laboratory (HDL) of the Universidad de los Andes, based on the vertical gap data mentioned in Tab. 1. The pilot consists of six independent wooden crates, five of which would be the "train" and the remaining one would correspond to the "platform". It is important to mention that the platform box is of utmost importance, since it contains the force plate, so its design had to be special, meticulous, and more complex. The decision to build the drawers independently was made because this would solve the problem that they would generate undesired movements while the participants were walking on them in the experiment. Once the pilot was assembled, it was fastened together with four rubber bands, generating a single rigid structure with the five drawers corresponding to the "train". In this way, the plate could be moved to each different vertical gap level easily and effectively. It should be noted that the design of the box corresponding to the platform was the most important of this study since any failure would generate noise in the data. This is due to the sensitivity of the force plate during the measurements. A solid and heavy base of wooden planks was made for the force plate so that the weight would help prevent vibrations and would lock separately to the box.

The scenarios to simulate passenger alighting from the train to the platform are shown in Fig. 3, which values are in the range of observations of Santiago subway stations reported in Tab. 1. The dimensions of the platform were fixed for all scenarios: width of $0.80 \mathrm{~m}$ and length of $1.50 \mathrm{~m}$. However, the vertical gap changed for each scenario: $0 \mathrm{~cm}$, $3.75 \mathrm{~cm}, 7.5 \mathrm{~cm}, 11.25 \mathrm{~cm}$ and $15 \mathrm{~cm}$.

Due to the pandemic (COVID 19), it was not possible to recruit passengers with reduced mobility for the experiment. This was because the tests were carried out during the months when the city of Santiago de Chile was under quarantine measures and with 


\begin{tabular}{lll}
\hline Station name (Line number) & Vertical gap - Direction 1 [cm] & Vertical gap - Direction 2 [cm] \\
\hline Central Station (L1) & 1.5 & 1.7 \\
Pedro de Valdivia (L1) & 0 & 1.3 \\
Los Dominicos (L1) & 1.5 & 1.7 \\
Vespucio Norte (L2) & 12.5 & 1.8 \\
Parque O'Higgins (L2) & 10.5 & 1.7 \\
La Cisterna (L2) & 1.7 & 1.7 \\
Puente Cal y Canto (L3) & 6.0 & 6.0 \\
Irarrazaval (L3) & 5.5 & 5.3 \\
Plaza Egana (L3) & 4.5 & 5.5 \\
Tobalaba (L4) & 6.3 & 4.2 \\
Quilin (L4) & 2.7 and 4.0 & 3.0 and 5.0 \\
Hospital Sotero del Rio (L4) & 2.2 & 4.7 \\
La Cisterna (L4A) & 6.5 & 4.0 \\
Santa Rosa (L4A) & 4.7 & 4.0 \\
Vicuna Mackenna (L4A) & 1.7 & 1.7 \\
Blanqueado (L5) & 11.0 & 9.0 \\
Nuble (L5) & 6.6 & 8.0 \\
Vicente Valdes (L5) & 6.0 & 1.6 \\
Lo Valledor (L6) & 5.3 & 5.0 \\
Franklin (L6) & 5.5 & 5.5 \\
Los Leones (L6) & 5.0 & 5.0 \\
\hline
\end{tabular}

Table 1 Vertical gap dimensions observed in subway stations in Santiago de Chile 

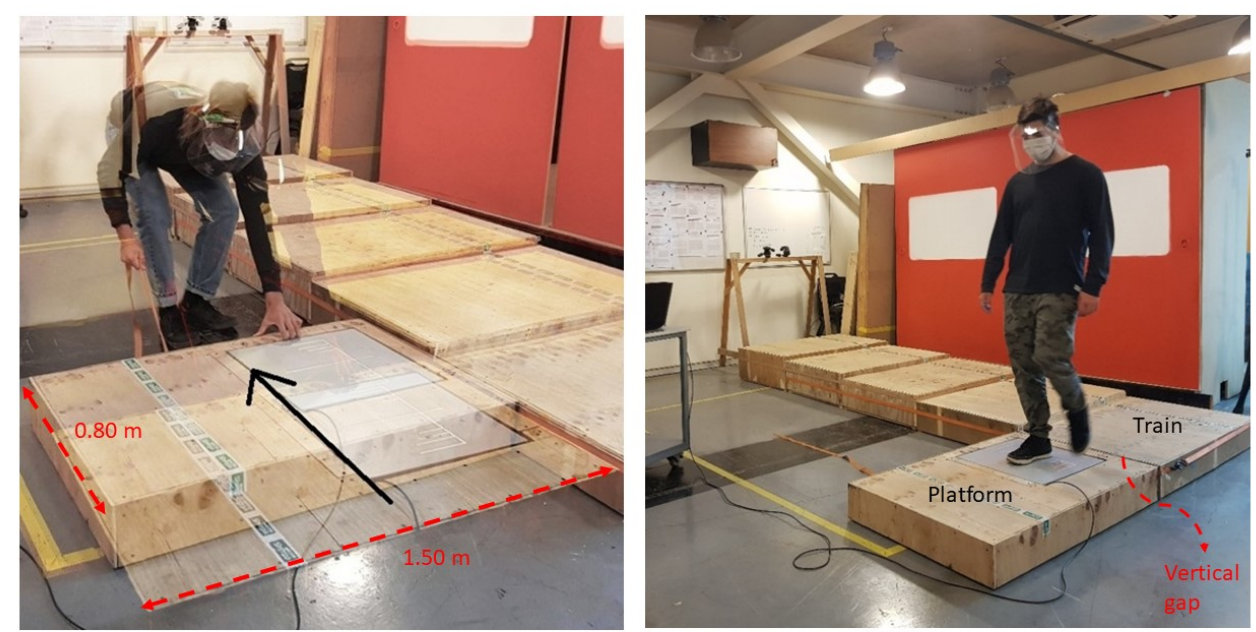

Figure 3 Mock-up at the Human Dynamics Laboratory: "platform" drawer movement for different vertical gaps (left), passenger stepping on the force plate (right)

a high number of active cases. Therefore, only participants with unrestricted health conditions participated in the study. The individual impairment was simulated through cases as a reduction in their mobility. The participants of the experiment were five young men aged $26 \pm 2$ years old, among them a height range of $172.4 \pm 7.7 \mathrm{~cm}$, with a body mass of $70.4 \pm 9.7 \mathrm{~kg}$. The exclusion criteria for participants (inability to participate) were inability to follow instructions, cardiac instability conditions, lower limb joint replacement, arthritis, diabetes, vision problems, or any other neuromuscular problem that may prevent participants from being able to execute the trial safely and effectively. Participants could not be on a prescription medication within the last seven days and had no experience of walking abnormalities within the last three years. All participants reported having a dominant right foot, which they define as the preferred leg for kicking a soccer ball. With the latter, we can reduce errors in the results by ensuring that the person used his or her right foot to walk down from the train to the platform [28].

Regarding the scenarios, four case studies were considered (see Fig. 4) for each vertical gap situation defined in Fig. 3. In each case, we simulated characteristics in the participants by adding a certain factor of reduced mobility. In each case, the gait process, i.e. the alighting of the train to the platform, was faced. To give greater validity to the data, three repetitions were performed for each trial. In summary, five participants were considered in four cases, with five different vertical gaps, in the alighting process considering three repetitions per case.

The four cases were defined following the study of Wang and Gillette [23]. In the first case (Case 1) each participant experimented without factors affecting their mobility. This case was considered as a baseline measurement to be compared with the values obtained by the other cases. On the other hand, Case 2 is defined when each member wore an orthopedic boot on the right foot, which gave them less mobility, trying to simulate a 

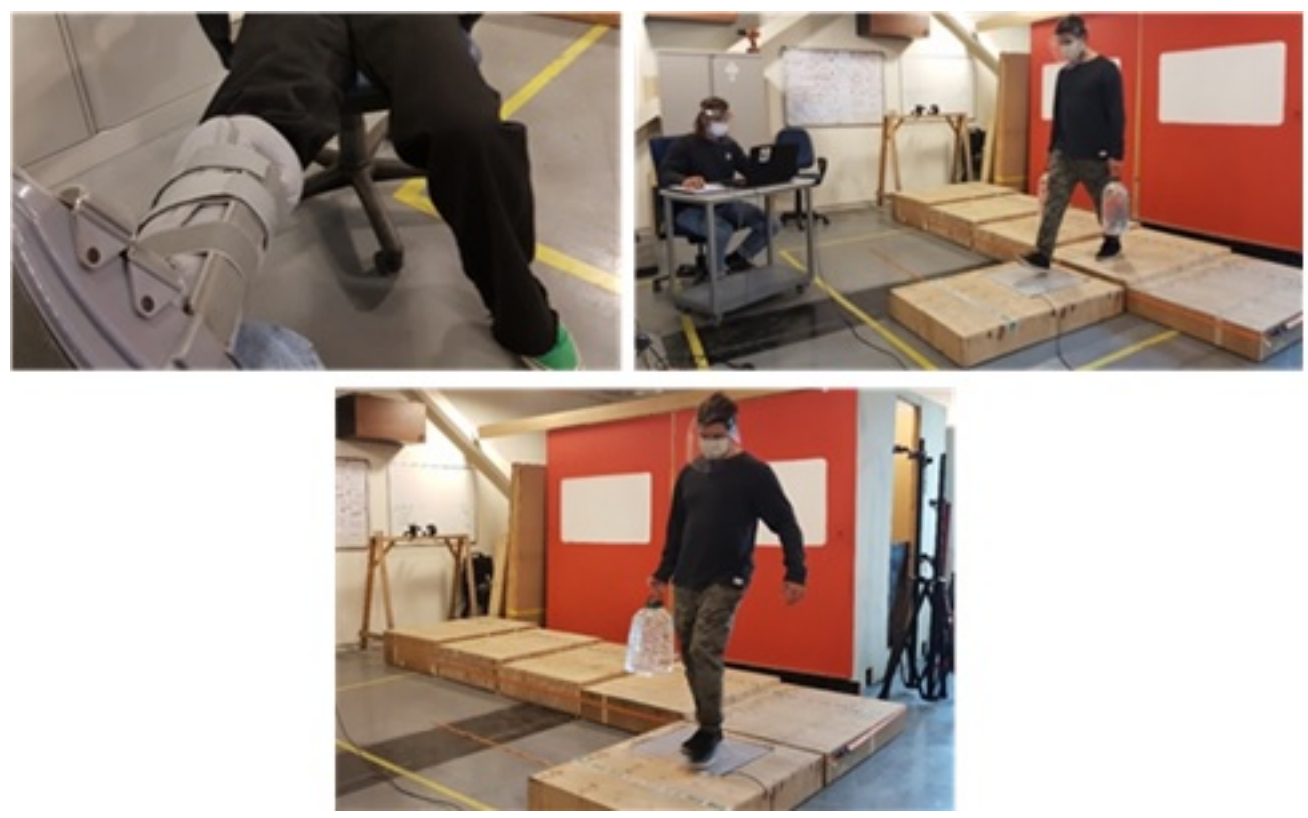

Figure 4 Cases to be considered in laboratory experiments: orthopedic boot (upper left), symmetric loading (upper right), asymmetric loading (lower)

person with problems or injuries in their lower extremities. In Case 3 each member carried $20 \%$ of his body mass symmetrically distributed in his right and left hand, to simulate a passenger with two shopping bags. Similarly, in Case 4 each member carried $20 \%$ of their body mass distributed asymmetrically in their right hand, seeking to simulate a passenger with a single, heavier-loaded shopping bag.

\subsection{Variable measurements}

The Bertec software was used to obtain the data from the plate which was installed in the mock-up. The software itself is simple and user-friendly for working with the force plate. A frequency of $100 \mathrm{~Hz}$ was used regarding the data storage. One of the most important characteristics of the software is that it has a digital auto-zero filter, which made it possible to eliminate any additional noise produced by vibrations in the environment. The value used as a filter was $4 \mathrm{~kg}$ or $39.2 \mathrm{~N}$ in the $\mathrm{z}$-axis, which is recommended by the developer and is set by default. By using the filter all points associated with values less than $4 \mathrm{~kg}$ in the z-axis were eliminated, and therefore these eliminated values did not affect the area and velocity of the actual gait process and decreases the dispersion of the results. Using different Python libraries, a code was generated to obtain values for each gait process. Therefore, the code was placed in the same folder as all the other files. It automatically read each step value and generated matrices associated with the name of each file. These matrices were of dimensions $(3, n)$, where $n$ is the length of the file, the first column is the force on the z-axis (Fx Z), while the second column is the anteroposterior plane and the third column is the lateral midplane. In summary, the variables obtained for each file were the area of the ellipse $95 \%$ confidence $\left(\mathrm{cm}^{2}\right)$, the anteroposterior movement length $(\mathrm{cm})$, 
the lateral midplane motion length $(\mathrm{cm})$, the anteroposterior movement velocity $(\mathrm{cm} / \mathrm{s})$, the lateral plane movement velocity $(\mathrm{cm} / \mathrm{s})$, and the sum of both velocities squared $(\mathrm{cm} / \mathrm{s})$.

Once all the files had been read by the code, the variables obtained from each one were transferred to an Excel spreadsheet where the three repetitions made in each vertical gap and case for the five participants were averaged. Once the spreadsheet was completed, the data was exported in Power-BI, which is a platform that allows operations to be performed and visualized appropriately. In this way, we were able to study how the stability of the participants variables in each case of the experiment when changing the vertical gaps. These obtained variables were combined by obtaining the following variables compared per case (from now on VC). This comparison is given because no single variable by itself can give us much information, but by seeing how they vary from one another we can obtain useful information. The VC1 is defined as the ratio between the area of the ellipse and the lenght of the foot. VC1 is of great help to study the behavior of the area formed by the $95 \%$ confidence ellipse. The longer the length of the foot, the greater the anteroposterior plane path, and therefore the greater the area of the ellipse. By making this division, it is possible to arrive at a value where the physical characteristics of the participant's foot do not affect the data. The lateral midplane represents stability. The greater the lateral midplane, the less stability during the gait process facing a vertical gap. When comparing different vertical gaps, the anteroposterior range of motion will vary, and therefore it is necessary to compare it with the lateral midplane in a ratio. In this sense, VC2 is defined as the division between the lateral midplane and the anteroposterior plane. Another division is needed to compare how large was the velocity of the footfall in the anteroposterior plane compared to the anteroposterior range of motion (VC3). If this value increases, stability decreases. The lower the anteroposterior range, the lower the angle of inclination in the anteroposterior plane. This angle is the one used to enter and exit the gait process. When the participant is feeling instability before a vertical gap, the foot seeks to enter with an angle of inclination closer to 90 degrees. Therefore, there is no less anteroposterior range of motion. Furthermore, if the anteroposterior range is carried out at an equal or greater velocity, it could be caused because the person seeks to exit the situation quickly to achieve stability before falling. This is commonly seen in people who stumble when descending from a train or who cannot control their velocity in this process.

A last VC is defined (VC4), which has the previous logic of VC3. However, in this case VC4 specify the relation of the velocity vector that is composed by the square root of the sum of the velocity of the footfall in the anteroposterior plane squared and lateral midplane squared, which is then divided by the area of the ellipse.

Comparison variables VC2, VC3, and VC4 are divisions of variables with different units. These VCs are not exposed by their resulting unit since they do not have a physical sense. Rather, they are presented as divisions since the aim is to see how they behave with each other, and whether their relationship increases or decreases as the vertical gaps vary. The VCs are compared using box plots, which are used for groups of scaled scores. This way, it allows studying the distribution characteristics of a group of scores, as well as the level of the scores. The scores are ordered from lowest to highest, and four groups of equal size are formed from the ordered scores. That is, $25 \%$ of all scores are placed in 
each group. The lines dividing the groups are called quartiles, and the groups are called quartile groups. Generally, these groups are labeled from 1 to 4 starting at the bottom. In addition, a circular dot was added to the results to give the average of the data, so that a trend of the data can be seen, which adds to the understanding of the data. Each point corresponds to the average of the three repetitions made by each participant.

\section{Results}

\subsection{Case 1 (baseline)}

The results of this case (without factors affecting the mobility of participants) are of great importance since they are the reference for comparison of the rest of the cases. Fig. 5 shows that VC 1 (ratio of the ellipse area to the foot length) shows a slight but steady decrease as the vertical gap increases. We expected to see an increase around the ellipse since with increasing vertical the participants should have presented a negative change in their stability. In fact, this did happen, however, four of the five participants changed their gait strategy when faced with larger vertical gap. To change the strategy, the angle of inclination in the anteroposterior plane must be decreased. That is, the right foot sought to be in a position closer to $90^{\circ}$ when reaching the platform. Although in this study it was not possible to measure the angle of inclination, we can deduce whether this variable decreased or increased by relating the other $\mathrm{VC}$ of the base case.
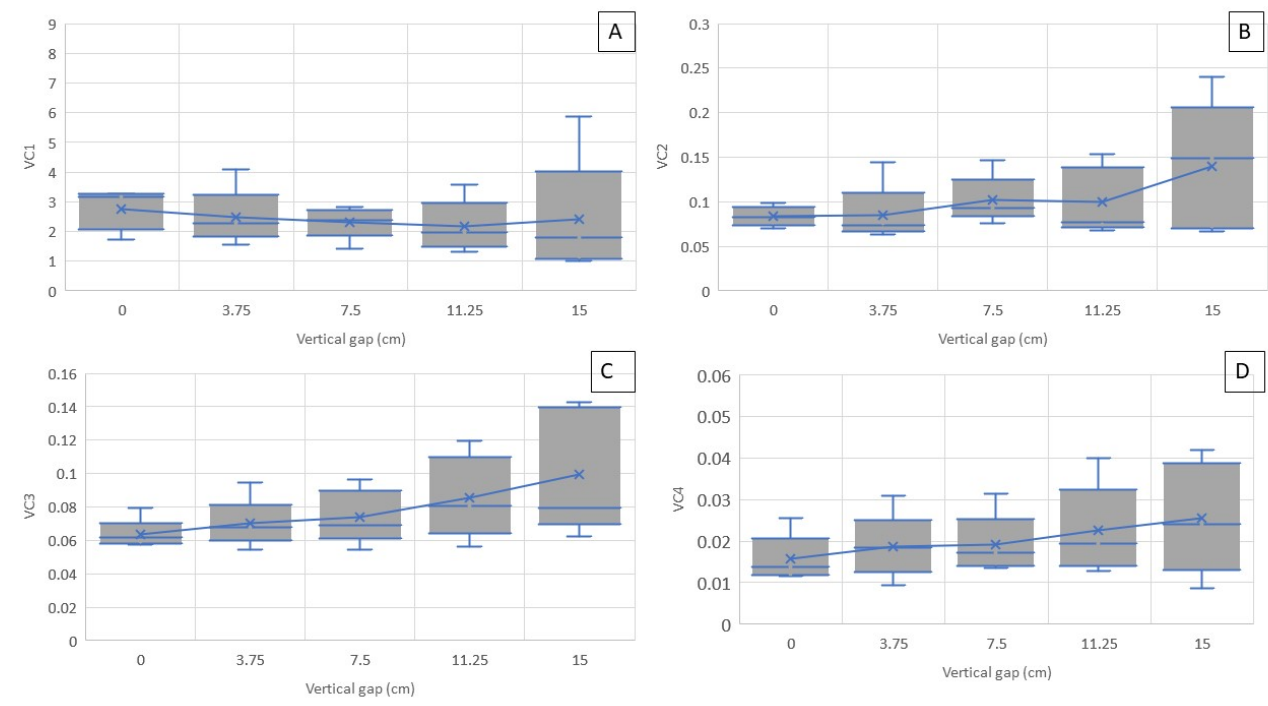

Figure 5 VC for each vertical gap in Case 1 (baseline): A) VC1, B) VC2, C) VC3, D) VC4

Regarding VC2 (ratio of the lateral midplane to the anteroposterior plane) Fig. 6 shows a constant but small increase in instability. This means that the lateral midplane stability was negatively affected by increasing the vertical gap. The biggest change can be seen between the vertical gap of $11.25 \mathrm{~cm}$ and $15 \mathrm{~cm}$. In the case of VC3 (ratio between the anteroposterior plane and the anteroposterior motion velocity) and VC4 (ratio 
between the anteroposterior motion velocity, the lateral midplane motion velocity, and the ellipse area), Fig. 5 shows a clear trend of increasing instability (i.e. a greater vertical gap produced an increase in the VC4). This proves that the angle of inclination of the anteroposterior plane decreased steadily with increasing the vertical gap. As explained above, this is because the participants changed their gait strategy. The graphs obtained for Case 1 were used as the basis for comparison in the following cases. It is emphasized that all the VCs behaved as expected, except for VC1. In this one we expected to see growth instead of a decrease, however, when analyzing it and seeing how the rest of VCs behaved, we can understand that the reason is related to the change of the gait strategy. It can be concluded from Case 1 that the higher the vertical gap, the greater the instability, where a vertical gap of $15 \mathrm{~cm}$ was the most detrimental to the participants.

\subsection{Case comparison}

Wearing an orthopedic boot (Case 2) did not result in a greater instability when increasing the vertical gap. The values on average in Fig. 6 remained without variations. This means something in the orthopedic boot provided some help to the participants. A possible reason for this small variation in the values obtained for each VC is because the sole of the boot is curved, which affects the anteroposterior plane and lateral medial plane. This curvature means that the center of pressure tends to be centered throughout the entire stride process, decreasing its anteroposterior plane and lateral midplane ranges, and therefore the VC did not show a clear tendency of greater instability as the vertical gaps increased. This does not mean that this increased instability did not occur, but rather that the method employed based on the center of pressure, failed to measure well the treads (footfall) with this type of sole with curvature.
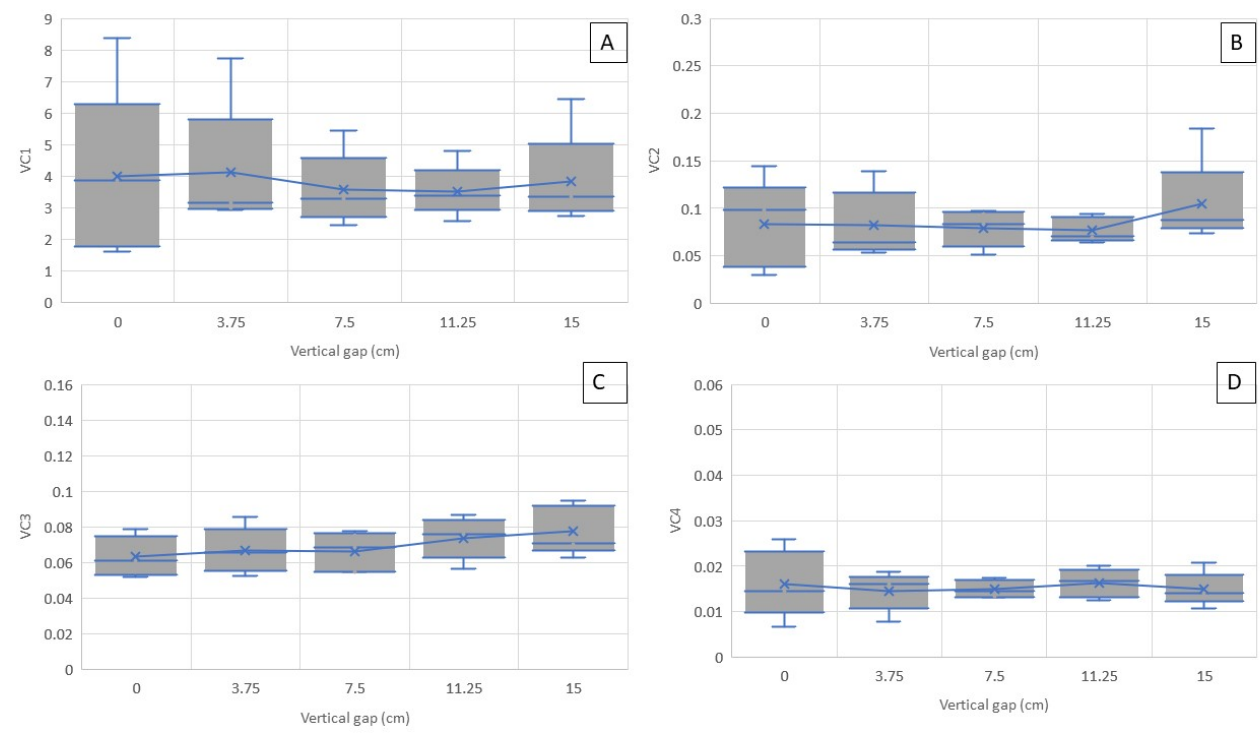

Figure 6 VC for each vertical gap in Case 2 (orthopedic boot): A) VC1, B) VC2, C) VC3, D) VC4 

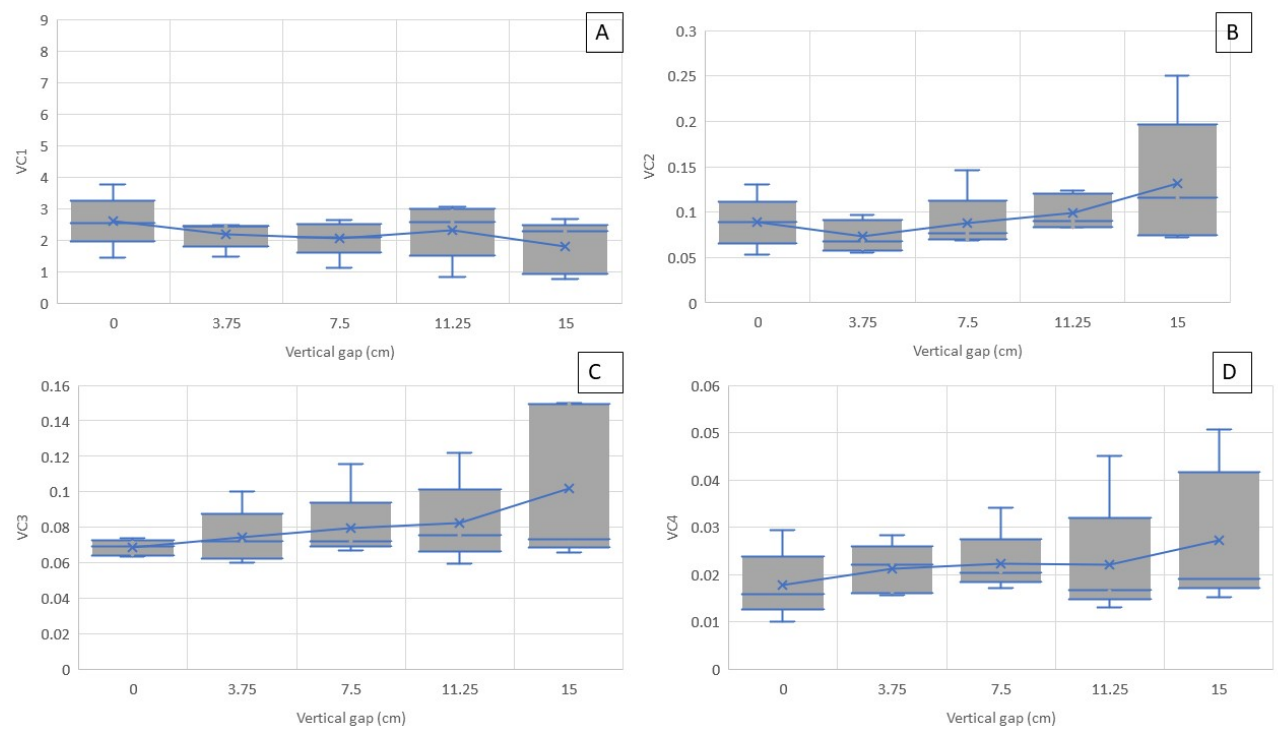

Figure 7 VC for each vertical gap in Case 3 (symmetrical loading): A) VC1, B) VC2, C) VC3, D) VC4
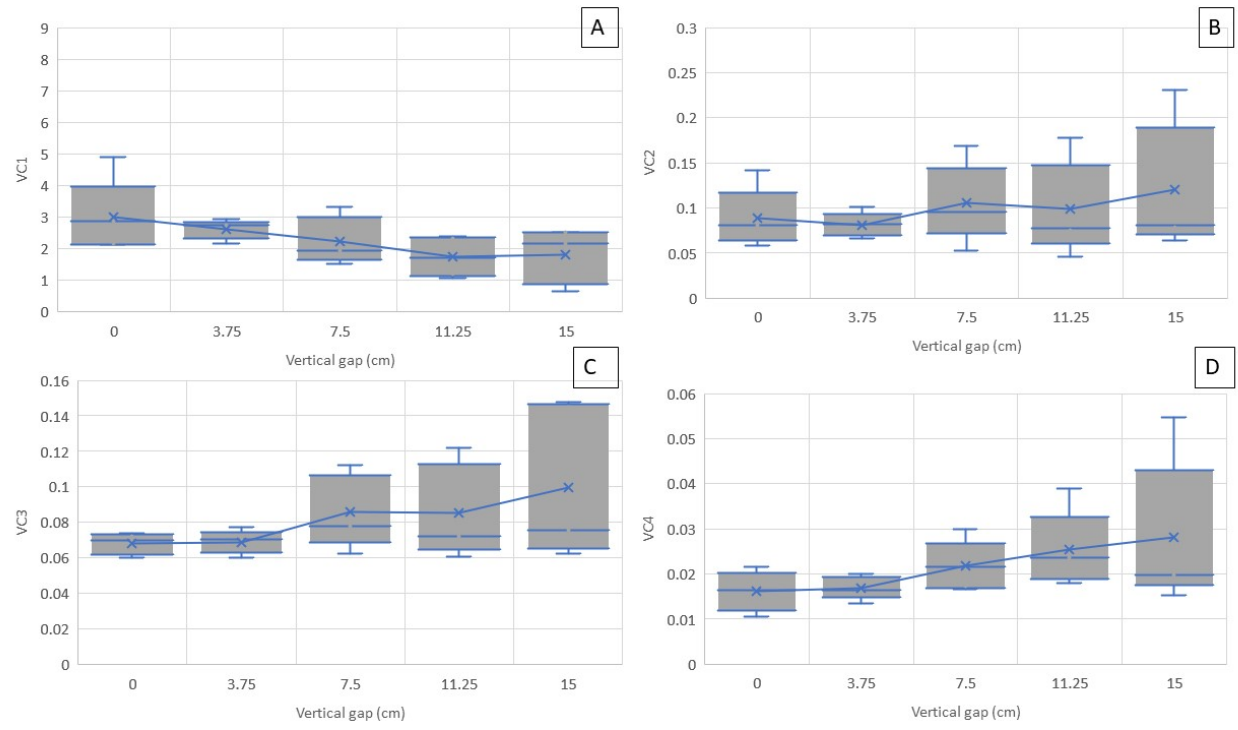

Figure 8 VC for each vertical gap in Case 4 (asymmetrical loading): A) VC1, B) VC2, C) VC3, D) VC4

Fig. 7 shows that Case 3 (symmetrical loading), is a more unstable version compared to Case 1. This is due to the extra weight added to the participants which corresponded to $20 \%$ of their weight. This instability became noticeable in the last vertical gap, which has a clear explanation because the higher the vertical gap the weight carried by each participant generates an acceleration, and therefore the participants had to make a greater effort to remain stable (reflected especially in VC2 and VC3).

In Case 4 (asymmetrical loading) Fig. 8 shows similar trends to Case 3. VC2 shows an increase similar to Case 3, implying that the lateral midplane stability was negatively affected by increasing the vertical gap. Surprisingly, the variation was less than those of 

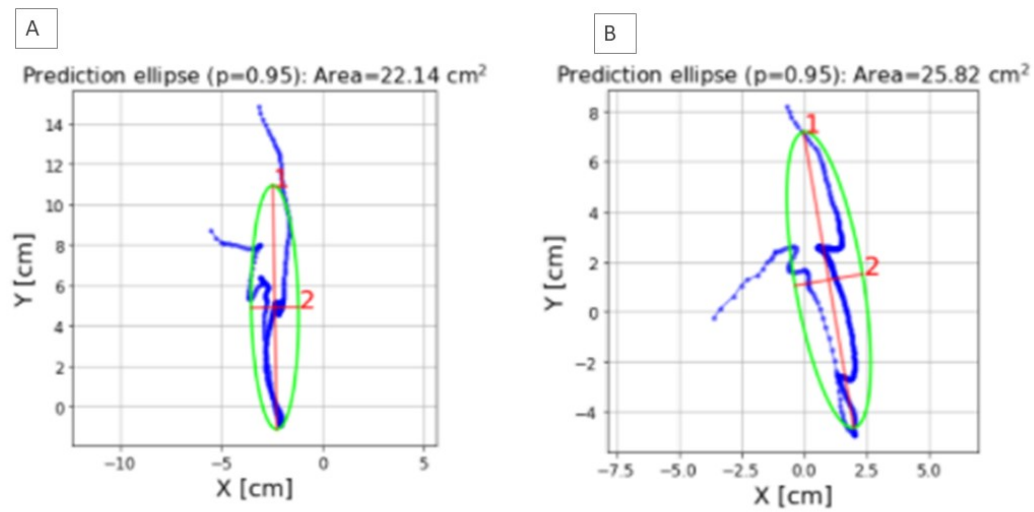

Figure 9 A) V-shaped displacement of the center of pressure in Case 3. B) V-shaped displacement of the center of pressure in Case 4

Case 1 and Case 3. However, a new phenomenon was observed (also in Case 3) during visual inspection of the tread charts.

This phenomenon was shown in erratic and abnormal center of pressure displacements in $3.5 \%$ of files corresponding to a vertical gap of $15 \mathrm{~cm}$. This phenomenon was also observed for a vertical gap of $11.25 \mathrm{~cm}$. The participants as shown in Fig. 9, presented a "V" shaped displacement (line blue in Fig. 9) instead of a linear displacement. The instability generated was so high that the damping was done first with the toes. This phenomenon is considered common in a jumping process, but not in a gait process using steps. Then, the weight shifted to the heels to continue with the gait process.

\section{Conclusions}

This article focused on studying the variables that affect the gait and stability of passengers with reduced mobility when facing different vertical gaps. To this, the methodology was based on laboratory experiments that could determine an effective way to measure these variables in the process of alighting from an urban train to a platform. The experiments included a mock-up of a train and its adjacent platform. In the experiments a force plate was installed to measure the center of pressure displacement, and therefore the anteroposterior plane (lenght and velocity), the lateral midplane (length and velocity) and the area of the $95 \%$ confidence ellipse were obtained. Different compared variables (VCs) were defined to analyze the 4 different cases for 5 vertical gap scenarios (performing 3 repetitions per case).

The 4 different study cases, allowed us to give a sample of data to study the impact of vertical gaps on the gait and stability of passengers with reduced mobility. Case 1 (baseline) provided more information since the results were the points of comparison 
for the rest of the cases. It turns out that the area of the ellipse is not the best way to measure instability, since the participants of the experiment took changes in the gait strategy for different vertical gaps, which generated a change in the angle of inclination of the anteroposterior plane of their stride.

In Case 2 the curved sole of the orthopedic boot did not allow the center of pressure displacement to be measured correctly. This makes sense since a curved sole makes the person less likely to fall or slip and thus counteracts the negative effect of this boot on mobility. Orthopedic boots are used in case of injuries or fractures produced in the lower extremities and unlike clinical casts, these can be removed, to help the patient. Their construction is mostly made of high-density plastic and padded fabric so as not to cause injury to the wearer's skin. These boots restrict full mobility in the calf, ankle, and toes, resulting in difficulty walking.

Cases 3 and 4, gave a new phenomenon. They provided greater instability data than Case 1, and also showed erratic displacements for a vertical gap of 1.25 and $15 \mathrm{~cm}$. This shows that a vertical gap greater than $15 \mathrm{~cm}$ is not suitable for passengers with reduced mobility.

One of the areas that this study was not able to delve into, and could be a source of inspiration for future research is to make simultaneous center of pressure and center of mass measurements on the participants as reported in Lee and Chou [18]. In this way, it would be possible to obtain the angle of inclination in the anteroposterior plane and lateral midplane, obtaining empirical data of their values during a gait process, which will replace the method of the ellipse area. Here, a more complex measurement system should be used, which would add a necessary amount of time to carry out the experiment. However, this path is recommended since this article is rather exploratory, and starts from the basics, so a study that expands on the experimentation performed would be an excellent complement to this line of study.

On the other hand, further studies should consider a larger number of participants that could be gathered to be part of the experiment at the laboratory facility. This is limited to the framework of the COVID19 pandemic, which is going through the country and the world at the moment. Future experiments will also consider the effect of the vertical gap on the gait and instability for those passengers who are boarding the train.

It is concluded that the results provided to determine the change of gait process in passengers with reduced mobility considering different vertical gaps, gives evidence that the higher the vertical gap, the more instability is generated directly and indirectly. Although it was not possible to find a final design or to provide absolute values of vertical gaps, the results allow comparing scenarios and cases, which show that it is not possible to have ranges greater than $15 \mathrm{~cm}$ in healthy people (Case 1) and ranges greater than $11.25 \mathrm{~cm}$ in passengers with reduced mobility since this would cause high instability in the gait process when descending from an urban train to the platform.

Acknowledgements The authors would like to thank those who participated in the experiments at the Human Dynamics Laboratory of the Universidad de los Andes. This study is supported by ANID (Chile) FONDECYT Project 11200012 which was approved by the Scientific Ethics Committee of the Universidad de los Andes (Chile). 


\section{References}

[1] Transport Subsecretary: Plan Maestro 2025. Ministry of Transport and Telecommunications, Chile, (In Spanish), (2013)

[2] Ministry of Housing and Urbanization: Universal Accessibility Law 20.422, (In Spanish), (2010)

[3] Ministry of Housing and Urbanization: Decreto Supremo 50, (In Spanish), (2016)

[4] Tyler, N.: Accessibility and the Bus System. Thomas Telford, United Kingdom, (2002)

[5] Alonso, F.: Algo más que suprimir barreras: conceptos y argumentos para una accesibilidad universal. TRANS, Revista de Traductología, 11, 15-30, (In Spanish), (2007)

[6] Marquez, L., Poveda, J. C., Vega, L. A.: Factors affecting personal autonomy and perceived accessibility of people with mobility impairments in an urban transportation choice context. Journal of Transport \& Health, 14, 100583, (2019)

[7] Bovonsunthonchai, S., Ariyaudomkit, R., Susilo, T. E., Sangiamwong, P., Puchaphan, P., Chandee, S., Richards, J.: The impact of different mobile phone tasks on gait behaviour in healthy young adults. Journal of Transport \& Health, 19, 100920, (2020)

[8] Seriani, S., Fernández, R.: Planning guidelines for metro-bus interchanges by means of a pedestrian microsimulation model. Transportation Planning and Technology, 38(5), 569-583, (2015)

[9] Seriani, S., Fernández, R., Oyanedel, C.: The impact of gap dimensions on passengers boarding and alighting using different mobility aids at railway stations by laboratory experiments. International Journal of Rail Transportation, 9(2), 186-202, (2021)

[10] Fernández, R., Zegers, P., Weber, G., Tyler, N.: Influence of platform height, door width, and fare collection on bus dwell time: laboratory evidence for Santiago de Chile. Transportation Research Record, 2143(1), 59-66, (2010)

[11] Fernández, R., Valencia, A., Seriani, S.: On passenger saturation flow in public transport doors. Transportation Research Part A: Policy and Practice 78, 102-112, (2015)

[12] Holloway, C., Thoreau, R., Roan, T-R., Boampong, D., Clarke, T., Watts, D.: Effect of vertical step height on boarding and alighting time of train passengers. In: Proceedings of the Institution of Mechanical Engineers Part F Journal of Rail and Rapid Transit 230(4), 1234-1241 (2016) 
[13] Daamen, W., Lee, Y., Wiggenraad, P.: Boarding and alighting experiments: an overview of the set up and performance and some preliminary results on the gap effects. Transportation Research Record 2042, 71-81 (2008)

[14] Atkins: Significant Steps, Research commissioned by UK Department for Transport, (2004), London

[15] Tyler, N., Childs, C., Boampong, D., Fujiyama, T.: Investigating ramp gradients for humps on railway platforms. Municipal Engineer 168(2), 150-160 (2015)

[16] Startzell, J. K., Owens, D. A., Mulfinger, L. M., Cavanagh, P. R., 2000. Stair negotiation in older people: a review. Journal of the American Geriatrics Society, 48(5), 567-580, (2000)

[17] Hashish, R., Toney-Bolger, M. E., Sharpe, S. S., Lester, B. D., Mulliken, A.: Texting during stair negotiation and implications for fall risk. Gait \& Posture, 58, 409-414, (2017)

[18] Lee, H. J., Chou, L. S.: Detection of gait instability using the center of mass and center of pressure inclination angles. Archives of Physical Medicine and Rehabilitation, 87(4), 569-575, (2006)

[19] Bhatt, T., Espy, D., Yang, F., Pai, Y. C.:Dynamic gait stability, clinical correlates, and prognosis of falls among community-dwelling older adults. Archives of Physical Medicine and Rehabilitation 92(5), 799-805, (2011)

[20] Song, Q., Sun, W., Zhang, C., Mao, M., Li, L.:Effects of a Dual-Task Paradigm and Gait Velocity on Dynamic Gait Stability during Stair Descent. Applied Sciences, 10(6), 1979, (2020),

[21] Anker, L. C., Weerdesteyn, V., Van Nes, I. J., Nienhuis, B., Straatman, H., Geurts, A. C.: The relation between postural stability and weight distribution in healthy subjects. Gait \& Posture, 27(3), 471-477, (2008)

[22] Vieira, M. F., Lehnen, G. C., Noll, M., Rodrigues, F. B., De Avelar, I. S., Da Costa, P. H. L.: Use of a backpack alters gait initiation of high school students. Journal of Electromyography and Kinesiology,28, 82-89, (2016)

[23] Wang, J., Gillette, J. C.: Mediolateral postural stability when carrying asymmetric loads during stair negotiation. Applied Ergonomics, 85, 103057, (2020)

[24] Huang, M. H., Brown, S. H.: Age differences in the control of postural stability during reaching tasks. Gait \& Posture, 38(4), 837-842, (2013)

[25] Lafond, D., Corriveau, H., Hébert, R., Prince, F.: Intrasession reliability of center of pressure measures of postural steadiness in healthy elderly people. Archives of Physical Medicine and Rehabilitation, 85(6), 896-901, (2004) 
[26] Zatsiorsky, V. M., Duarte, M.: Rambling and trembling in quiet standing. Motor Control, 4(2), 185-200, (2000)

[27] Witt, A., Kurths, J., Pikovsky, A.: Testing stationarity in time series. Physical Review E, 58(2), 1800, (1998)

[28] Gribble, P. A., Hertel, J., Denegar, C. R.:Chronic ankle instability and fatigue create proximal joint alterations during performance of the Star Excursion Balance Test. International Journal of Sports Medicine, 28(03), 236-242, (2007) 